\title{
ON THE SCOPE OF AVERAGING FOR FRANKL'S CONJECTURE
}

\author{
GÁBOR CZÉDLI, MIKLÓS MARÓTI, AND E. TAMÁS SCHMIDT
}

\begin{abstract}
Let $\mathcal{F}$ be a union-closed family of subsets of an $m$-element set $A$. Let $n=|\mathcal{F}| \geq 2$. For $b \in A$ let $w(b)$ denote the number of sets in $\mathcal{F}$ containing $b$ minus the number of sets in $\mathcal{F}$ not containing $b$. Frankl's conjecture from 1979, also known as the union-closed sets conjecture, states that there exists an element $b \in A$ with $w(b) \geq 0$.

The present paper deals with the average of the $w(b)$, computed over all $b \in A$. $\mathcal{F}$ is said to satisfy the averaged Frankl's property if this average is nonnegative. Although this much stronger property does not hold for all unionclosed families, the first author [7] verified the averaged Frankl's property whenever $n \geq 2^{m}-2^{m / 2}$ and $m \geq 3$.

The main result of this paper shows that (1) we cannot replace $2^{m / 2}$ with the upper integer part of $2^{m} / 3$, and (2) if Frankl's conjecture is true (at least for $m$-element base sets) and $n \geq 2^{m}-\left\lfloor 2^{m} / 3\right\rfloor$ then the averaged Frankl's property holds (i.e., $2^{m / 2}$ can be replaced with the lower integer part of $2^{m} / 3$ ). The proof combines elementary facts from combinatorics and lattice theory. The paper is self-contained, and the reader is assumed to be familiar neither with lattices nor with combinatorics.
\end{abstract}

\section{Introduction AND THE MAIN THEOREM}

Given an $m$-element finite set $A=\left\{a_{1}, \ldots, a_{m}\right\}$, a family (or, in other words, a set) $\mathcal{F}$ of subsets of $A$, i.e. $\mathcal{F} \subseteq P(A)$, is called a union-closed family (over A) if $X \cup Y \in \mathcal{F}$ whenever $X, Y \in \mathcal{F}$. We always assume that $A$ is finite with $3 \leq m:=|A|$ and $n:=|\mathcal{F}| \geq 2$. It was Peter Frankl in 1979 who formulated the following conjecture, now known as Frankl's conjecture or the union-closed sets conjecture: if $\mathcal{F}$ is as above then there exists an element of $A$ which is contained in at least half of the members of $\mathcal{F}$. In spite of at least three dozen papers, this conjecture is still open. Hence it will be convenient to use the following terminology: we say that Frankl's conjecture holds over m-element base sets, if for any unionclosed family $\mathcal{F}$ of subsets of an $m$-element (equivalently, at most $m$-element) set $A$ with $|\mathcal{F}| \geq 2$, there exists an element of $A$ which is contained in at least half of the members of $\mathcal{F}$.

Clearly, it is sufficient to consider only those union-closed sets that contain the empty set. Hence in the sequel, when the size $|\mathcal{F}|$ of $\mathcal{F}$ will be important, we will always assume that $\emptyset \in \mathcal{F}$.

Date: January 8, 2008, revised: December 11, 2008.

Key words and phrases. Union-closed sets, Frankl's conjecture, lattice.

This research was partially supported by the NFSR of Hungary (OTKA), grant no. T 049433, T 48809 and K 60148. 
The known achievements on Frankl's conjecture belong to two categories. The first category is constituted by those (in fact, the majority of) results that belong to pure combinatorics, with respect to both the statements and their proofs. There are several directions and the titles of the listed references speak for themselves, so we mention only a few results relevant to our investigations. Bošnjak and Marković [5] prove that Frankl's conjecture holds over eleven-element base sets, while Roberts [30] settles the case $n=|\mathcal{F}| \leq 40$ and $n<4 m-1$. As an opposite to Roberts' result on "small families", Gao and $\mathrm{Yu}[13]$ verify the conjecture for "very large families", i.e. for those with

$$
n \geq 2^{m}-12\left(\frac{3}{2}\right)^{[m / 3]}-\frac{1}{2}\left(\begin{array}{c}
m \\
3
\end{array}\right)-\frac{5}{3} m+44.5 .
$$

For other achievements of combinatorial nature cf., e.g., Norton and Sarvate [21] and Vaughan [32]. One can read more about the problem at [37] or, of course, in Frankl [12].

On the other hand, Stanley [31] and Poonen [22] establish a nice lattice theoretic version of Frankl's conjecture. (For details one can also see [7] or Abe and Nakano [3].) This initiated a series of lattice theoretical papers given by Abe and Nakano [1], [2], [3], [4], Herrmann and Langsdorf [14], and Reinhold [24] (some of which contained results already known in the folklore); these are the results belonging to the second category.

However, there were no real links between the combinatorial and the lattice theoretical approaches before [7], except of course for the statement of their equivalence. In particular, results that look "combinatorial" were proved by combinatorial methods, and the lattice theoretical results have not had a significant influence on combinatorists. This is very surprising, for the lattice theoretic approach has at least one obvious advantage: while it is fairly difficult to visualize a union-closed family with, say, $(m, n)=(5,12)$, depicting the Hasse diagram of the corresponding twelve element lattice creates no problem at all.

Probably, [7] is the first case when a purely combinatorial statement is proved within lattice theory. Similarly, the present paper belongs to neither of the abovementioned two categories. Our main result is purely combinatorial without mentioning lattices. Its proof is a mixture of lattice theory and combinatorics. However, only the rudiments of lattice theory and those of combinatorics are used. So the paper is intended to be self-contained for most of the readers.

Let $\mathcal{F}$ be a union-closed family over $A$ and let the notations $n=|\mathcal{F}| \geq 2$, $m=|A|=\left\{a_{1}, \ldots, a_{m}\right\}$ be fixed throughout. For $a \in A$ let

$$
w(a)=|\{X \in \mathcal{F}: a \in X\}|-|\{X \in \mathcal{F}: a \notin X\}| .
$$

Then Frankl's conjecture claims the existence of an $a \in A$ with $w(a) \geq 0$. With the notation

$$
\overline{\mathbf{w}}(\mathcal{F})=\frac{1}{|A|} \sum_{a \in A} w(a)
$$

let us say that $\mathcal{F}$ satisfies the averaged Frankl's property if

$$
\overline{\mathbf{w}}(\mathcal{F}) \geq 0 .
$$

Although this property clearly implies that Frankl's conjecture holds for the given $\mathcal{F}$, it belongs to the folklore that many union-closed families fail to satisfy the averaged Frankl's property. 
For a given $m=|A|$, the maximum value of $n$ is of course $2^{m}$. For "large" union-closed families $\mathcal{F}$ including $\emptyset$, it is proved in [7] that

$$
|\mathcal{F}| \geq 2^{m}-2^{m / 2} \Longrightarrow \overline{\mathbf{w}}(\mathcal{F}) \geq 0 .
$$

This statement is much stronger than Gao and Yu's (1) in two senses: (3) covers many more instances of $\mathcal{F}$, and "Frankl's" is replaced by "averaged Frankl's".

Even if $2^{m / 2}$ is better, i.e., larger, than the corresponding expression in (1), already [7] observes that $2^{m / 2}$ is not the optimal value. The original target of the present paper was to replace $2^{m / 2}$ in (3) with the best possible value, in the additional hope that the improved version of (3) gives more information on the original Frankl's conjecture as well. Unfortunately, this extra hope is not fulfilled yet, for the main theorem below assumes the validity of Frankl's conjecture. As usual, the upper resp. lower integer part of a real number $x$ will be denoted by $\lceil x\rceil$ resp. $\lfloor x\rfloor$; for example $\lceil 32 / 3\rceil=11$.

Main Theorem. Let $m \geq 3$, and let $A$ be an m-element set.

(1) There exists a union-closed family $\mathcal{F}$ over $A$ with $\emptyset \in \mathcal{F}$ and $|\mathcal{F}|=\left\lfloor 2^{m+1} / 3\right\rfloor=$ $\left\lceil 2^{m+1} / 3\right\rceil-1$ such that $\mathcal{F}$ fails the averaged Frankl's property.

(2) Assume that Frankl's conjecture holds over m-element base sets. Then each union-closed family $\mathcal{F}$ over $A$ with $\emptyset \in \mathcal{F}$ and

$$
n:=|\mathcal{F}| \geq\left\lceil 2^{m+1} / 3\right\rceil
$$

satisfies the averaged Frankl's property.

Frankl's conjecture has been intensively studied and it is almost three decades old. Hence it is reasonable to conjecture that (4) in itself implies $\overline{\mathbf{w}}(\mathcal{F}) \geq 0$ for union-closed families $\mathcal{F}$.

The rest of the paper is devoted to the proof of this theorem and will run as follows. First we introduce some integer sequences, and study their elementary properties. This requires only elementary arguments with induction. Then we study order-ideals and (order-) semi-ideals (to be defined later) of finite Boolean lattices. We are interested in how to maximize the sum (equivalently, the average) of heights of elements of an order-ideal or semi-ideal $X$ when $|X|$ is fixed. Using the properties of our integer sequences we will show the expected but nontrivial fact that this maximum is available via the obvious greedy algorithm. Order-ideals do not create an invincible problem; however, our treatment for semi-ideals needs the assumption that Frankl's conjecture holds over $m$-element base sets. This leads to a new conjecture, formulated at the end of the paper.

Once the greedy algorithm for semi-ideals is proven to be appropriate, the Main Theorem follows immediately from its obvious reformulation for semi-ideals. Although this reformulation translates the problem to Lattice Theory, this is an easier and less elegant translation than the usual one by Stanley [31] and Poonen [22].

\section{Some integer SEQuences}

We are going to define and study three kinds of integer sequences: $\vec{\alpha}, \vec{\beta}$ and $\vec{\gamma}$. The $\vec{\alpha}$ resp. $\vec{\beta}$ sequences calculate the total height of greedy order-ideals resp. greedy semi-ideals (to be defined later). The $\vec{\gamma}$ sequences are the stepping stone between 
these two kinds of ideals, and the $\vec{\gamma}$ sequences are also needed to understand the inner structure of $\vec{\beta}$ sequences.

In this section, $m$ and $n$ will denote arbitrary natural numbers. Given two integer sequences $\vec{a}=\left(a_{1}, \ldots, a_{m}\right)$ and $\vec{b}=\left(b_{1}, \ldots, b_{n}\right)$, their concatenation will be denoted by

$$
\left(a_{1}, \ldots, a_{m}\right) \uplus\left(b_{1}, \ldots, b_{n}\right)=\left(a_{1}, \ldots, a_{m}, b_{1}, \ldots, b_{n}\right) .
$$

The length of a sequence $\vec{a}$ is denoted by $\operatorname{length}(\vec{a})$; it is always a positive integer and we have $\operatorname{length}(\vec{a})+\operatorname{length}(\vec{b})=\operatorname{length}(\vec{a} \uplus \vec{b})$. When $\operatorname{length}(\vec{a})=\operatorname{length}(\vec{b})$ then $\vec{a}+\vec{b}$ and $\vec{a}-\vec{b}$ are understood componentwise, e.g.,

$$
\left(a_{1}, \ldots, a_{k}\right)+\left(b_{1}, \ldots, b_{k}\right)=\left(a_{1}+b_{1}, \ldots, a_{k}+b_{k}\right) .
$$

The constant sequence $(j, j, \ldots, j)$ will be denoted by $\bar{j}$; we use this notation only in connection with addition, so there will be no ambiguity what the length of $\bar{j}$ is. For example, $\left(a_{1}, \ldots, a_{k}\right)+\overline{1}$ is $\left(a_{1}+1, \ldots, a_{k}+1\right)$. When there exists a sequence $\vec{c}$ with $\vec{a} \uplus \vec{c}=\vec{b}$ then we say that $\vec{a}$ is a (proper) initial segment of $\vec{b}$. Now, via induction, let us define

$$
\begin{aligned}
& \vec{\alpha}^{(0)}=(0), \quad \vec{\alpha}^{(i+1)}=\vec{\alpha}^{(i)} \uplus\left(\overline{1}+\vec{\alpha}^{(i)}\right), \\
& \vec{\gamma}^{(i)}=\overline{1}+\vec{\alpha}^{(i)} \quad(i=0,1,2 \ldots), \text { and } \\
& \vec{\beta}^{(1)}=\gamma_{0}, \quad \vec{\beta}^{(i+1)}=\vec{\gamma}^{(i)} \uplus \vec{\beta}^{(i)}, \text { which means that } \\
& \vec{\beta}^{(j)}=\vec{\gamma}^{(j-1)} \uplus \vec{\gamma}^{(j-2)} \uplus \cdots \uplus \vec{\gamma}^{(1)} \uplus \vec{\gamma}^{(0)} \quad(j=1,2,3, \ldots) .
\end{aligned}
$$

For example,

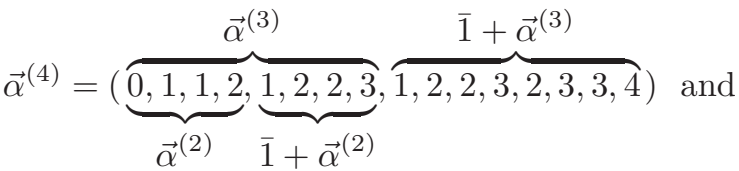

$$
\begin{aligned}
& \vec{\beta}^{(4)}=(\underbrace{1,2,2,3,2,3,3,4}_{\vec{\gamma}^{(3)}}, \underbrace{1,2,2,3}_{\vec{\gamma}^{(2)}}, \underbrace{1,2}_{\vec{\gamma}^{(1)}}, \underbrace{1}_{\vec{\gamma}^{(0)}}) .
\end{aligned}
$$

Notice that length $\left(\vec{\alpha}^{(i)}\right)=\operatorname{length}\left(\vec{\gamma}^{(i)}\right)=2^{i}(0 \leq i)$ while length $\left(\vec{\beta}^{(i)}\right)=2^{i}-1$ $(1 \leq i)$. The first member of a sequence is always indexed by 1 . The $i$ th member of $\vec{\alpha}^{(n)}$ will, of course, be denoted by $\vec{\alpha}_{i}^{(n)}$, and similar notations apply for $\vec{\beta}^{(n)}$ and $\vec{\gamma}^{(n)}$. For convenience, let $\vec{\alpha}^{(\infty)}$ resp. $\vec{\gamma}^{(\infty)}$ denote the infinite sequence whose initial segment of length $2^{k}$ is $\vec{\alpha}^{(k)}$ resp. $\vec{\gamma}^{(k)}$ for each $k \in \mathbf{N}=\{1,2, \ldots\}$.

For $0 \leq k \leq n$, the subsequences of the form

$$
\operatorname{segm}\left(\vec{a}, 2^{k}, i\right):=\left(a_{i 2^{k}+1}, a_{i 2^{k}+2}, \ldots, a_{(i+1) 2^{k}}\right), \quad i=0,1, \ldots, 2^{n-k}-1
$$

are called $2^{k}$-segments of $\vec{a}=\left(a_{1}, \ldots, a_{2^{n}}\right)$. Consecutive $2^{k}$-segments will play an important role in the forthcoming considerations. Let

$$
\begin{aligned}
& \operatorname{segm}\left(\vec{a}, 2^{k}, i\right), \ldots, \operatorname{segm}\left(\vec{a}, 2^{k}, i+\ell-1\right) \text { and } \\
& \operatorname{segm}\left(\vec{a}, 2^{k}, j\right), \ldots, \operatorname{segm}\left(\vec{a}, 2^{k}, j+\ell-1\right)
\end{aligned}
$$

be two families of $\ell$ consecutive $2^{k}$-segments, and consider two subsets $X$ and $Y$ of the corresponding index sets, i.e., let $X \subseteq\left\{t: i 2^{k}+1 \leq t \leq(i+\ell) 2^{k}\right\}$ and $Y \subseteq\left\{t: j 2^{k}+1 \leq t \leq(j+\ell) 2^{k}\right\}$. We say that $X$ and $Y$ are equally positioned in 
these families of $2^{k}$-segments if $X \rightarrow Y, x \mapsto x+(j-i) 2^{k}$ is a bijection. That is, "equally positioned" has the natural meaning.

Given a sequence $\vec{a}=\left(a_{1}, \ldots, a_{\ell}\right)$; the sum of $t$ consecutive members of $\vec{a}$ beginning at the $i$ th position will be denoted by

$$
\sigma(\vec{a}, i, t)=a_{i}+a_{i+1}+\cdots+a_{i+t-1} .
$$

This notation assumes that $1 \leq i, 0 \leq t$ and $i+t-1 \leq \ell$. Notice that $\sigma(\vec{a}, i, 0)=0$ by convention. The forthcoming lemmas will be formulated only for $\vec{\alpha}^{(n)}$, but other than Lemma 2 they will be obviously valid and used for $\vec{\gamma}^{(n)}$ as well. As usual, $\mathbf{N}_{0}=\mathbf{N} \cup\{0\}$ denotes the set of nonnegative integers.

Lemma 1. Let $i \in \mathbf{N}$ and $j \in \mathbf{N}_{0}$ with $i+j-1 \leq 2^{n}$. Then $\sigma\left(\vec{\alpha}^{(n)}, 1, j\right) \leq$ $\sigma\left(\vec{\alpha}^{(n)}, i, j\right)$.

Proof. We can assume that $i>1$. We use induction on $j$. Since $\vec{\alpha}_{1}^{(n)}$ is the only occurrence of 0 in $\vec{\alpha}^{(n)}$, the statement is evident for $j \leq 1$. So we assume that $j>1$ and the lemma holds for $1, \ldots, j-1$. For brevity, let $x=\sigma\left(\vec{\alpha}^{(n)}, 1, j\right)$ and $y=\sigma\left(\vec{\alpha}^{(n)}, i, j\right)$.

If $i \leq j$ (pictorially: if $x$ and $y$ overlap) then

$$
\begin{aligned}
& x=\sigma\left(\vec{\alpha}^{(n)}, 1, i-1\right)+\sigma\left(\vec{\alpha}^{(n)}, i, j-i+1\right) \text { and } \\
& y=\sigma\left(\vec{\alpha}^{(n)}, i, j-i+1\right)+\sigma\left(\vec{\alpha}^{(n)}, j+1, i-1\right),
\end{aligned}
$$

and $x \leq y$ follows from the induction hypothesis.

Hence we can assume that $i>j$. The pictorial illustration below (even if it does not reflect the full generality) will be useful for what comes next:

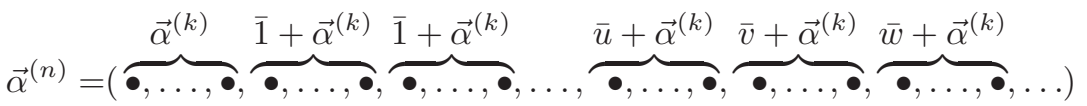

$$
\begin{aligned}
& \vec{\alpha}^{(n)}=(\bullet, \ldots, \underbrace{\bullet, \bullet, \ldots, \bullet, \bullet}_{z}, \ldots, \bullet, \ldots, \bullet, \ldots, \underbrace{\bullet, \bullet, \ldots, \bullet, \bullet}_{y}, \ldots, \bullet, \ldots) .
\end{aligned}
$$

Consider the unique $k \in \mathbf{N}_{0}$ such that $2^{k} \leq j<2^{k+1}$. The assumption that $x$ and $y$ do not overlap implies that $k \leq n-1$.

Firstly, we assume that $k<n-1$. Then we can choose three consecutive $2^{k}$ segments, say $\operatorname{segm}\left(\vec{\alpha}^{(n)}, 2^{k}, q\right), \operatorname{segm}\left(\vec{\alpha}^{(n)}, 2^{k}, q+1\right)$ and $\operatorname{segm}\left(\vec{\alpha}^{(n)}, 2^{k}, q+2\right)$, such that the summands of $y$ belong to this family $B$ of consecutive $2^{k}$-segments. (More precisely but less pictorially, such that $q 2^{k}+1 \leq i$ and $i+j-1 \leq(q+3) 2^{k}$.) Consider also the family $A$ of the $2^{k}$-segments $\operatorname{segm}\left(\vec{\alpha}^{(n)}, 2^{k}, 0\right), \operatorname{segm}\left(\vec{\alpha}^{(n)}, 2^{k}, 1\right)$ and $\operatorname{segm}\left(\vec{\alpha}^{(n)}, 2^{k}, 2\right)$, that is, the first three $2^{k}$-segments. Let $z$ be the sum of $j$ consecutive members in the first three $2^{k}$-segments such that the summands of $z$ in $A$ and the summands of $y$ in $B$ are equally positioned. (More formally, $i=q 2^{k}+r$ and $z=\sigma\left(\vec{\alpha}^{(n)}, r, j\right)$.) Observe that $A$ consists of $\vec{\alpha}^{(k)}, \overline{1}+\vec{\alpha}^{(k)}, \overline{1}+\vec{\alpha}^{(k)}$ while $B$ consists of $\bar{u}+\vec{\alpha}^{(k)}, \bar{v}+\vec{\alpha}^{(k)}, \bar{w}+\vec{\alpha}^{(k)}$ for appropriate positive integers $u, v, w$.

Now, $0<u, 1 \leq v$ and $1 \leq w$, whence the elements of the first three $2^{k}$ segments are less than or equal to the corresponding elements of $\operatorname{segm}\left(\vec{\alpha}^{(n)}, 2^{k}, q\right)$, $\operatorname{segm}\left(\vec{\alpha}^{(n)}, 2^{k}, q+1\right)$, and $\operatorname{segm}\left(\vec{\alpha}^{(n)}, 2^{k}, q+2\right)$. Hence $z \leq y$, for $z$ and $y$ are equally positioned. Further, $x \leq z$ follows from the previously considered "overlapping" case, and we conclude $x \leq y$. 
Secondly, we assume that $k=n-1$. Then $\vec{\alpha}^{(n)}$ consists of two $2^{k}$-segments only. Since $x$ and $y$ do not overlap, we obtain that $j=2^{k}$ and $i=2^{k}+1$. Using $\vec{\alpha}^{(n)}=$ $\vec{\alpha}^{(k)} \uplus\left(\overline{1}+\vec{\alpha}^{(k)}\right)$, we conclude that $x=\sigma\left(\vec{\alpha}^{(k)}, 1,2^{k}\right)<\sigma\left(\overline{1}+\vec{\alpha}^{(k)}, 1,2^{k}\right)=y$.

Define the inverse of a sequence $\vec{a}=\left(a_{1}, a_{2}, \ldots, a_{k}\right)$ as

$$
\operatorname{inv}\left(a_{1}, a_{2}, \ldots, a_{k}\right)=\left(a_{k}, a_{k-1}, \ldots, a_{1}\right) .
$$

The proof, a trivial induction, of the following lemma is left to the reader.

Lemma 2. $\vec{\alpha}^{(n)}+\operatorname{inv}\left(\vec{\alpha}^{(n)}\right)=\bar{n}$.

Now we formulate a statement on the sum of the last $j$ members of $\vec{\alpha}^{(n)}$.

Lemma 3. If $j \leq 2^{n}$ and $1 \leq i<2^{n}-j$ then $\sigma\left(\vec{\alpha}^{(n)}, i, j\right) \leq \sigma\left(\vec{\alpha}^{(n)}, 2^{n}-j+1, j\right)$.

Proof. Consider the sequence $\bar{n}-\vec{\alpha}^{(n)}$. This sequence is the inverse of $\vec{\alpha}^{(n)}$, so the sum of the last $j$ members becomes the sum of the first $j$ members. Hence the assertion follows from Lemma 1.

Lemma 4. Let $i, j<2^{n}$ and define

$$
u:=\left\{\begin{array}{ll}
i+j, & \text { if } i+j \leq 2^{n} \\
2^{n}, & \text { if } i+j>2^{n}
\end{array} \quad \text { and } \quad v:=i+j-u .\right.
$$

Then $\sigma\left(\vec{\alpha}^{(n)}, 1, i\right)+\sigma\left(\vec{\alpha}^{(n)}, 1, j\right) \leq \sigma\left(\vec{\alpha}^{(n)}, 1, u\right)+\sigma\left(\vec{\alpha}^{(n)}, 1, v\right)$.

Proof. If $i+j \leq 2^{n}$ then $v=0$ gives $\sigma\left(\vec{\alpha}^{(n)}, 1, v\right)=0$, and the assertion is a trivial consequence of Lemma 1 . Hence we can assume that $i+j>2^{n}$. Let us compute; the application of Lemma 3 will be denoted by ${ }^{*}$, and we will use that $j-v=2^{n}-i$ and (therefore) $j>v$ :

$$
\begin{gathered}
\sigma\left(\vec{\alpha}^{(n)}, 1, i\right)+\sigma\left(\vec{\alpha}^{(n)}, 1, j\right)=\sigma\left(\vec{\alpha}^{(n)}, 1, i\right)+\sigma\left(\vec{\alpha}^{(n)}, 1, v\right)+\sigma\left(\vec{\alpha}^{(n)}, v+1, j-v\right) \\
\leq * \sigma\left(\vec{\alpha}^{(n)}, 1, i\right)+\sigma\left(\vec{\alpha}^{(n)}, 1, v\right)+\sigma\left(\vec{\alpha}^{(n)}, i+1,2^{n}-i\right)= \\
\sigma\left(\vec{\alpha}^{(n)}, 1,2^{n}\right)+\sigma\left(\vec{\alpha}^{(n)}, 1, v\right)=\sigma\left(\vec{\alpha}^{(n)}, 1, u\right)+\sigma\left(\vec{\alpha}^{(n)}, 1, v\right) .
\end{gathered}
$$

Lemma 5. If $1 \leq i<2^{n}$ then $\vec{\beta}_{i}^{(n)} \leq \vec{\gamma}_{i}^{(n)}$.

Proof. An easy induction on $n$. If $i \leq 2^{n-1}$ then $\vec{\beta}_{i}^{(n)}=\vec{\gamma}_{i}^{(n)}$ by definition. Otherwise $\vec{\beta}_{i}^{(n)}=\vec{\beta}_{i-2^{n-1}}^{(n-1)} \leq \vec{\gamma}_{i-2^{n-1}}^{(n-1)}<\left(\overline{1}+\vec{\gamma}^{(n-1)}\right)_{i-2^{n-1}}=\vec{\gamma}_{i}^{(n)}$.

Lemma 6. If $0 \leq j<2^{n-1}$ and $0 \leq i \leq 2^{n-1}$ then $\sigma\left(\vec{\beta}^{(n)}, 2^{n-1}+1, j\right) \leq$ $\sigma\left(\vec{\beta}^{(n)}, i, j\right)$.

Proof. For brevity, let $x=\sigma\left(\vec{\beta}^{(n)}, 2^{n-1}+1, j\right)$ and $y=\sigma\left(\vec{\beta}^{(n)}, i, j\right)$.

First assume that $i+j-1 \leq 2^{n-1}$. This means that the summands of $y$ lie entirely in $\vec{\gamma}^{(n-1)}$; however, the following illustration is only a particular case (for $y$ and $z$ may overlap):

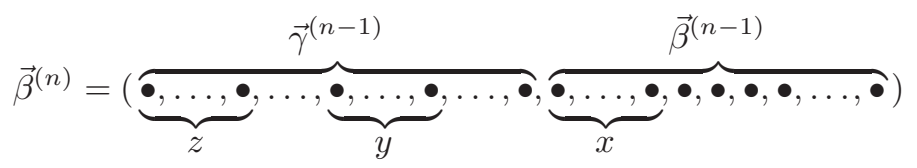

For $z=\sigma\left(\vec{\beta}^{(n)}, 1, j\right)=\sigma\left(\vec{\gamma}^{(n-1)}, 1, j\right)$ we obtain $x \leq z$ from Lemma 5 . Then $z \leq \sigma\left(\vec{\gamma}^{(n-1)}, i, j\right)=y$ by Lemma 1 , whence $x \leq y$. 
Now we assume that $2^{n-1}<i+j-1$, which means that $x$ and $y$ overlap. Let $z=\sigma\left(\vec{\beta}^{(n)}, 2^{n-1}+1, i+j-2^{n-1}-1\right)$, the "intersection of $x$ and $y$ ", $u=$ $\sigma\left(\vec{\beta}^{(n)}, i+j, 2^{n-1}+1-i\right), v=\sigma\left(\vec{\beta}^{(n)}, i, 2^{n-1}+1-i\right)$, and, further, let $w=$ $\sigma\left(\vec{\beta}^{(n)}, i+j-2^{n-1}, 2^{n-1}+1-i\right)$. Then $x=z+u$ and $y=z+v$, cf. the illustration below (notice that $w$ and $v$ may overlap).

$$
\vec{\beta}^{(n)}=(\overbrace{\bullet, \bullet, \ldots, \bullet, \underbrace{\bullet, \ldots, \bullet}_{w}, \ldots, \underbrace{\vec{\gamma}^{(n-1)}, \ldots, \bullet}_{v}}^{\overbrace{\underbrace{\bullet \bullet, \ldots, \bullet}_{v}}^{\underbrace{\bullet, \ldots, \bullet}_{z}, \ldots, \bullet, \ldots, \bullet}} \underbrace{\vec{\beta}^{(n-1)}}_{u})
$$

Since $u$ and $w$ are "equally positioned", Lemma 5 gives $u \leq w$. Then Lemma 3 applied to $\vec{\gamma}^{(n-1)}$ yields $w \leq v$. Finally, $u \leq v$ implies $x=z+u \leq z+v=y$.

Lemma 7. If $0 \leq i<2^{n-1}$ and $0 \leq j \leq 2^{n-1}$ then

$$
\sigma\left(\vec{\beta}^{(n-1)}, 1, i\right)+\sigma\left(\vec{\gamma}^{(n-1)}, 1, j\right) \leq \sigma\left(\vec{\beta}^{(n)}, 1, i+j\right) .
$$

Proof. Since $\vec{\beta}^{(n)}=\vec{\gamma}^{(n-1)} \uplus \vec{\beta}^{(n-1)}$, we can compute:

$$
\begin{gathered}
\sigma\left(\vec{\beta}^{(n-1)}, 1, i\right)+\sigma\left(\vec{\gamma}^{(n-1)}, 1, j\right)=\sigma\left(\vec{\beta}^{(n)}, 2^{n-1}+1, i\right)+\sigma\left(\vec{\beta}^{(n)}, 1, j\right) \leq^{*} \\
\sigma\left(\vec{\beta}^{(n)}, j+1, i\right)+\sigma\left(\vec{\beta}^{(n)}, 1, j\right)=\sigma\left(\vec{\beta}^{(n)}, 1, i+j\right),
\end{gathered}
$$

where ${ }^{*}$ stands for an application of Lemma 6 .

For a sequence $\vec{a}$, let $\overline{\mathbf{E}}(\vec{a}, i, \ldots, j)$ denote

$$
\overline{\mathbf{E}}(\vec{a}, i, \ldots, j)=\frac{\sigma(\vec{a}, i, j-i+1)}{j-i+1}=\frac{a_{i}+a_{i+1}+\cdots+a_{j}}{j-i+1},
$$

the average of the elements in the segment $\left(a_{i}, a_{i+1}, \ldots, a_{j}\right)$. Remember that $\vec{\gamma}^{(\infty)}$ was introduced for convenience right before the definition of segments; of course $\vec{\gamma}^{(\infty)}$ could be replaced by $\vec{\gamma}^{(n)}$ in the following lemma.

Lemma 8. Let $2 \leq n \in \mathbf{N}$.

(1) For $k=1,2, \ldots,\left\lfloor 2^{n} / 3\right\rfloor, \overline{\mathbf{E}}\left(\vec{\beta}^{(n)}, 1, \ldots, k\right)=\overline{\mathbf{E}}\left(\vec{\gamma}^{(\infty)}, 1, \ldots, k\right) \leq n / 2$.

(2) $\overline{\mathbf{E}}\left(\vec{\beta}^{(n)}, 1, \ldots,\left\lfloor 2^{n} / 3\right\rfloor+1\right)=\overline{\mathbf{E}}\left(\vec{\gamma}^{(\infty)}, 1, \ldots,\left\lfloor 2^{n} / 3\right\rfloor+1\right)>n / 2$.

(3) For $\left\lfloor 2^{n} / 3\right\rfloor<k \leq 2^{n}-1, \overline{\mathbf{E}}\left(\vec{\beta}^{(n)}, 1, \ldots, k\right)>n / 2$.

Notice that the equations in Parts (1) and (2) are clear by definitions. Although Part (3) implies Part (2), we will prove only Parts (1) and (2). Part (3) will not be proved, for it will not be used in the sequel and its proof is similar to but considerably lengthier than the proofs of Parts (1) and (2).

Proof. Define

$$
S_{n}=\left\{k \in \mathbf{N}: \overline{\mathbf{E}}\left(\vec{\gamma}^{(\infty)}, 1, \ldots, t\right) \leq n / 2 \text { for } t=1,2, \ldots, k\right\} .
$$

One can easily see that proving that $\left\lfloor 2^{n} / 3\right\rfloor$ is the largest member of $S_{n}$ is equivalent to proving Parts (1) and (2) for $n$. We prove this via induction on $n$. The case $n=2$ is evident. Now suppose that $n \geq 3$ and that Parts (1) and (2) hold for $n-1$. 
The first few members of $\vec{\gamma}^{(\infty)}$ are depicted below for $n=5$ :

$$
\overbrace{\underbrace{\overbrace{\vec{\gamma}^{(n-3)}}^{\hat{1}_{1}+2,3, \vec{\gamma}^{(n-3)}}, \overbrace{2,3,3,4}^{\vec{\gamma}^{(n-1)}}}_{\vec{\gamma}^{(n-2)}}, \underbrace{2,3,3,4,3,4,4,5}_{\overline{1}+\vec{\gamma}^{(n-2)}}}^{\overline{1}+\vec{\gamma}^{(n-1)}}, \overbrace{2,3,3,4,3,4,4,5,3,4,4,5,4,5,5,6}^{\underbrace{}_{2}}, \ldots
$$

Since $2^{n-3} \leq\left\lfloor 2^{n-1} / 3\right\rfloor$, the induction hypothesis gives

$$
\overline{\mathbf{E}}\left(\vec{\gamma}^{(\infty)}, 1, \ldots, k\right) \leq(n-1) / 2 \text { for } 1 \leq k \leq 2^{n-3} .
$$

Hence $\left\{1,2, \ldots, 2^{n-3}\right\} \subseteq S_{n}$. It follows from Lemma 2 that

$$
\overline{\mathbf{E}}\left(\vec{\gamma}^{(\infty)}, 1, \ldots, 2^{n-3}\right)=(n-1) / 2 .
$$

Rewriting (6) from the first $2^{n-3}$-segment to the second one, which is $\overline{1}+\vec{\gamma}^{(n-3)}$, we obtain

$$
\overline{\mathbf{E}}\left(\vec{\gamma}^{(\infty)}, 2^{n-3}+1, \ldots, 2^{n-3}+k\right) \leq(n+1) / 2 \quad \text { for } 1 \leq k \leq 2^{n-3} .
$$

This implies $\left\{1,2, \ldots, 2^{n-2}\right\} \subseteq S_{n}$. Now, (7) for the second $2^{n-3}$-segment gives $\overline{\mathbf{E}}\left(\vec{\gamma}^{(\infty)}, 2^{n-3}+1, \ldots, 2^{n-3}+2^{n-3}\right)=(n+1) / 2$, which combined with (7) yields

$$
\overline{\mathbf{E}}\left(\vec{\gamma}^{(\infty)}, 1, \ldots, 2^{n-2}\right)=n / 2 \text {. }
$$

The shift from the first $2^{n-2}$-segment to the second one, $\overline{1}+\vec{\gamma}^{(n-2)}$, changes (8) into $\overline{\mathbf{E}}\left(\vec{\gamma}^{(\infty)}, 2^{n-2}+1, \ldots, 2^{n-1}\right)=1+n / 2=(n+2) / 2$, which together with (8) gives $\overline{\mathbf{E}}\left(\vec{\gamma}^{(\infty)}, 1, \ldots, 2^{n-1}\right)=(n+1) / 2$. Hence $2^{n-1} \notin S_{n}$, and we conclude that the largest member of $S_{n}$ is $2^{n-2}+k$ for some $0 \leq k<2^{n-2}$. It follows from (8) that $k$ is the largest number in $\left\{0,1, \ldots, 2^{n-2}-1\right\}$ such that $\overline{\mathbf{E}}\left(\vec{\gamma}^{(\infty)}, 2^{n-2}+\right.$ $\left.1, \ldots, 2^{n-2}+k\right) \leq n / 2$. Now going from the second $2^{n-2}$-segment $\overline{1}+\vec{\gamma}^{(n-2)}$ to the first $2^{n-2}$-segment $\vec{\gamma}^{(n-2)}$, we see that $k$ is the largest number in $\left\{0,1, \ldots, 2^{n-2}-1\right\}$ such that $\mathbf{E}\left(\vec{\gamma}^{(\infty)}, 1, \ldots, k\right) \leq n / 2-1=(n-2) / 2$. Hence $k$ is the largest member of $S_{n-2}$, whence the induction hypothesis gives $k=\left\lfloor 2^{n-2} / 3\right\rfloor$. Therefore, the largest member of $S_{n}$ is $2^{n-2}+\left\lfloor 2^{n-2} / 3\right\rfloor=\left\lfloor 2^{n-2}+2^{n-2} / 3\right\rfloor=\left\lfloor 2^{n} / 3\right\rfloor$.

\section{Semi-ideals and a Theorem equivalent to the Main Theorem}

By a lattice $(L ; \leq)$ we mean a partially ordered set such that for any $x, y \in L$ the supremum and infimum of $\{x, y\}$ exist; they are denoted by $x \vee y$ and $x \wedge y$, respectively. We deal only with finite lattices; they necessarily have a unique least element 0 and a unique largest element 1. For $a \leq b \in L$ the subset $\{x \in L: a \leq$ $x \leq b\}$ is denoted by $[a, b]$ and it is called an interval of $L$. When $a=0$ or $b=1$ then a particular notation applies: $\uparrow a=[a, 1]$ and $\downarrow b=[0, b]$. The covering relation $\prec$ is defined via $a \prec b$ iff $a \leq b$ and $|[a, b]|=2$. If $0 \prec a$ then $a$ is called an atom of the lattice.

Let $B_{m}$ denote the Boolean lattice of size $2^{m}$. Each $x \in B_{m}$ has a unique complement $x^{\prime}$ satisfying $x \vee x^{\prime}=1$ and $x \wedge x^{\prime}=0$. Notice that $B_{m}$ is isomorphic to the power set lattice over an $m$-element set; the singleton sets, the complements of subsets, the empty set and the whole set corresponding to the atoms, the complements of elements, 0 and 1 of $B_{m}$, respectively. The height $h(x)$ of an element $x \in B_{m}$ is the length of any maximal chain in $\downarrow x$. In the powerset model, the height is just the number of elements of the given subset. If $X$ is a subset of $B_{m}$, then the total 
height $h(X)$ of $X$ is defined to be the sum $\sum_{a \in X} h(a)$. If $X$ is a nonempty subset $X$ of $B_{m}$, then its average height is defined to be and denoted by

$$
\overline{\mathbf{h}}(X)=h(X) /|X| .
$$

A nonempty subset $X$ of $B_{m}$ is called an order-ideal if for any $x \in X, \downarrow x \subseteq X$. If a nonempty set $X$ is the union of certain intervals $\left[a_{i}, b_{i}\right]$ such that the $a_{i}$ are (not necessarily distinct) atoms, then $X$ is said to be a semi-ideal of $B_{m}$.

The goal of the next section is to show that whenever the size $|X|$ of an orderideal is fixed, then a straightforward greedy algorithm produces an order-ideal with maximum total height. We also prove the analogous statement for semi-ideals; however, we could not avoid assuming Frankl's conjecture in that case. The importance of semi-ideals is revealed by the following theorem and the lemma following it.

Theorem 1. Let $m \geq 3$.

(1) There exists a semi-ideal $X$ of $B_{m}$ such that $\overline{\mathbf{h}}(X)>m / 2$ and $|X|=$ $\left\lceil 2^{m} / 3\right\rceil$.

(2) Assume that Frankl's conjecture holds over m-element base sets. Then for each semi-ideal $X$ of $B_{m},|X| \leq\left\lfloor 2^{m} / 3\right\rfloor$ implies $\overline{\mathbf{h}}(X) \leq m / 2$.

By a proper subalgebra of $\left(B_{m}, \vee, 0\right)$ we mean a $\vee$-closed subset $Y$ of $B_{m}$ such that $0 \in Y \neq B_{m}$. The importance of the above theorem is due to

Lemma 9. Let $m \in \mathbf{N}$.

(1) For $X \subseteq B_{m}, X$ is a semi-ideal iff $B_{m} \backslash X$ is a proper subalgebra of $\left(B_{m}, \vee, 0\right)$.

(2) The Main Theorem and Theorem 1 are equivalent statements.

(3) Assume that Frankl's conjecture holds over m-element base sets. Then for each semi-ideal $X$ of $B_{m}$ other than $B_{m} \backslash\{0\},|X \cap \uparrow a| \leq|X| / 2$ for some atom $a \in B_{m}$.

Proof. Let $X$ be a semi-ideal, and let $u, v \in Y=B_{m} \backslash X$. By way of contradiction, suppose $u \vee v \notin Y$. Then $[p, u \vee v] \subseteq X$ for some atom $p \in B_{m}$. From $p \leq u \vee v$ we conclude $p \leq u$ or $p \leq v$, say, $p \leq u$, whence $u \in[p, u \vee v] \subseteq X$ contradicts $u \in Y$. This shows that $Y$ is a subalgebra of $\left(B_{m}, \vee, 0\right)$. It is proper, for $X \neq \emptyset$.

Now let $Y=B_{m} \backslash X$ be a proper subalgebra of $\left(B_{m}, \vee, 0\right)$. Then $X \neq \emptyset$. Let $u \in X$, we have to find an atom $p \in B_{m}$ with $[p, u] \cap Y=\emptyset$. Let $y$ denote the join of the set $Y \cap \downarrow u$. Since $y \in Y$, we have $y<u$ and therefore there is an atom $p \in \downarrow u \backslash \downarrow y$, which does the job. This proves Part (1).

Now, keeping formula (2) and the powerset model of $B_{m}$ in mind, for a proper subalgebra $F$ of $\left(B_{m}, \vee, 0\right)$, we let

$$
\overline{\mathbf{w}}(F)=\frac{1}{m} \cdot \sum_{a \text { is an atom of } B_{m}} w(a)
$$

where

$$
\begin{aligned}
w(a) & =|F \cap \uparrow a|-\left|F \cap \downarrow a^{\prime}\right|=2 \cdot|F \cap \uparrow a|-|F \cap \uparrow a|-\left|F \cap \downarrow a^{\prime}\right| \\
& =2 \cdot|F \cap \uparrow a|-|F| .
\end{aligned}
$$


Hence $\overline{\mathbf{w}}(F) \geq 0$ iff

$$
\begin{aligned}
0 & \leq \sum_{a \text { atom }}(2 \cdot|F \cap \uparrow a|-|F|)=2\left(-\sum_{a \text { atom }}|F| / 2+\sum_{a \text { atom }}|F \cap \uparrow a|\right) \\
& =2\left(-m|F| / 2+\sum_{(a, b): a \leq b \in F, a \text { atom }} 1\right) \\
& =2\left(-m|F| / 2+\sum_{b \in F} a \leq b, a \text { atom } 1\right) \\
& =2\left(-m|F| / 2+\sum_{b \in F} h(b)\right)=2(-m|F| / 2+h(F)) .
\end{aligned}
$$

Dividing by $2|F|$ we obtain that $\overline{\mathbf{w}}(F) \geq 0$ iff $\overline{\mathbf{h}}(F) \geq m / 2$. Now let $X=B_{m} \backslash F$, a semi-ideal by Part (1). Since $\overline{\mathbf{h}}\left(B_{m}\right)=m / 2, \overline{\mathbf{h}}(F) \geq m / 2$ iff $\overline{\mathbf{h}}(X) \leq m / 2$, completing the proof of Part (2).

Finally, the straightforward deduction of Part (3) from the above arguments is left to the reader.

\section{Greedy order-IDEAls and GReedy SEMI-IDEALS}

Given an integer $k \in\left\{1, \ldots, 2^{m}\right\}$, we are looking for a $k$-element order-ideal $X$ of the Boolean lattice $B_{m}$ with maximal total height $\overline{\mathbf{h}}(X)$. It is natural to construct $X$ in a greedy way by induction on $k$. For $k \leq 3$, every $k$-element order-ideal has the same total height. Suppose we know how to construct a $(k-1)$-element orderideal $Y$ of maximal total height. Then we let $X=Y \cup\{x\}$ such that $x \notin Y, X$ is an order-ideal and $h(x)$ is as large as possible. Now we have roughly described our greedy algorithm. The goal of this section is to show that it maximizes the total height, indeed. First of all, a more exact description of the greedy algorithm is necessary.

Let $a_{1}, a_{2}, \ldots, a_{m}$ be a fixed enumeration of the atoms of $B_{m}$. Associated with this enumeration we define a greedy enumeration of $B_{m}$ via induction as follows. (For $x, y \in B_{m}, x \triangleleft y$ will denote that $x$ precedes $y$ in the greedy enumeration.) For $m \leq 1$, let $\triangleleft$ coincide with the strict lattice order (which is a linear order, i.e., a chain, in this case). Now assume that $m>1$. Clearly, $a_{1} \vee \cdots \vee a_{m-1}$ is $a_{m}^{\prime}$, the complement of $a_{m}$. The isomorphism theorem of intervals yields that $a_{1} \vee a_{m}, \ldots, a_{m-1} \vee a_{m}$ is an enumeration of atoms of $\uparrow a_{m}$, which is a Boolean lattice. Let $\triangleleft_{2}$ denote the greedy enumeration of $\uparrow a_{m}$ associated with the mentioned enumeration of its atoms. Let $\triangleleft_{1}$ denote the greedy enumeration of $\downarrow a_{m}^{\prime}$ associated with the enumeration $a_{1}, \cdots, a_{m-1}$ of its atoms. Finally, let $\triangleleft$ be the "concatenation" of $\triangleleft_{1}$ and $\triangleleft_{2}$ in the following sense: for $x, y \in B_{m}, x \triangleleft y$ iff $x, y \in \downarrow a_{m}^{\prime}$ with $x \triangleleft_{1} y$, or $x, y \in \uparrow a_{m}$ with $x \triangleleft_{2} y$, or $x \in \downarrow a_{m}^{\prime}$ and $y \in \uparrow a_{m}$. The left-hand lattice in Figure 1 shows the greedy enumeration of $B_{3}$ associated with the "from left to right" enumeration (i.e., $a_{1}=c_{2}, a_{2}=c_{3}, a_{3}=c_{5}$ ) of its atoms. (The greedy enumeration is defined by the indexing: $c_{i} \triangleleft c_{j}$ iff $i<j$.) Now, by a greedy order-ideal of $B_{m}$ we mean a nonempty subset of the form $\left\{x \in B_{m}: x \unlhd b\right\}$ where $\triangleleft$ is a greedy enumeration of $B_{m}, x \unlhd b$ means $x \triangleleft b$ or $x=b$, and $b \in B_{m}$. In other words, the greedy order-ideals are the nonempty initial segments of greedy enumerations. The smallest greedy order-ideal is $\{0\}$ (take $b=0$ ) while the largest is $B_{m}$ (take $\left.b=1\right)$. The first part of the following lemma justifies our terminology. 

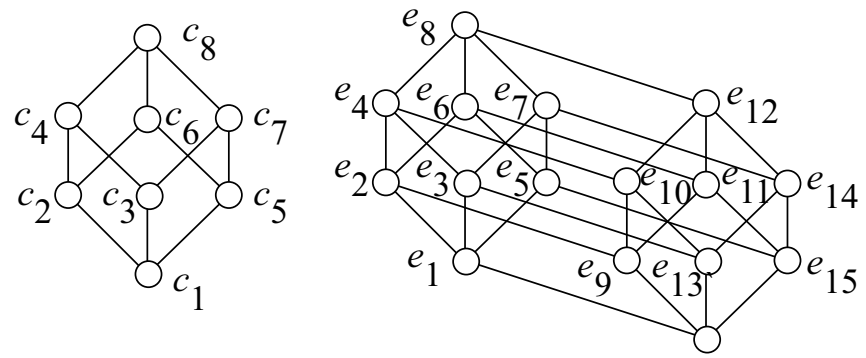

FIGURE 1

Lemma 10. Let $X$ be a greedy order-ideal of $B_{m}$. Then $X$ is an order-ideal of $B_{m}$, and its total height is

$$
h(X)=\sigma\left(\vec{\alpha}^{(m)}, 1,|X|\right) \text {, whence } \overline{\mathbf{h}}(X)=\overline{\mathbf{E}}\left(\vec{\alpha}^{(m)}, 1, \ldots,|X|\right) .
$$

The trivial induction proving this lemma is left to the reader. Now, let $a_{1}, a_{2}$, $\ldots, a_{m}$ still be a fixed enumeration of the atoms of $B_{m}$. For $j=1, \ldots, m$ we define the interval

$$
I_{j}=\left[a_{j}, a_{1}^{\prime} \wedge \cdots \wedge a_{j-1}^{\prime}\right] .
$$

In particular, $I_{1}=\left[a_{1}, 1\right] \cong B_{m-1}, I_{m}=\left[a_{m}, a_{1}^{\prime} \wedge \cdots \wedge a_{m-1}^{\prime}\right]=\left[a_{m}, a_{m}\right] \cong B_{0}$, and in general, $I_{j} \cong B_{m-j}$. In the right-hand lattice in Figure 1, if we start with the $a_{1}=e_{1}, a_{2}=e_{9}, a_{3}=e_{13}, a_{4}=e_{15}$ enumeration (i.e., from left to right enumeration) of atoms, we have $I_{1}=\left[e_{1}, e_{8}\right], I_{2}=\left[e_{9}, e_{12}\right], I_{3}=\left[e_{13}, e_{14}\right]$ and $I_{4}=\left\{e_{15}\right\}$. Notice that $\left\{I_{1}, \ldots, I_{m}\right\}$ is a partition of $B_{m} \backslash\{0\}$.

The sequence $I_{1}, I_{2}, \ldots, I_{m}$ is called a standard interval sequence of $B_{m}$; there are $m$ ! such sequences, for there are $m$ ! enumerations of the atoms. Now, by a greedy semi-ideal of $B_{m}$ we mean a nonempty subset $X$ of the form

$$
X=I_{1} \cup \cdots \cup I_{k-1} \cup U
$$

where $U$ is a greedy order-ideal of $I_{k}$. (Hence $U \neq \emptyset$ but $U=I_{k}$ is permitted.) The unique $k \in\{1, \ldots, m\}$ in (9) is said to be the rank of the greedy semi-ideal $X$, and it will be denoted by $\operatorname{rank}(X)$.

Lemma 11. Let $X \subseteq B_{m}$ be a greedy semi-ideal of $B_{m}$. Then it is indeed a semi-ideal, and its total height is

$$
h(X)=\sigma\left(\vec{\beta}^{(m)}, 1,|X|\right), \text { whence } \overline{\mathbf{h}}(X)=\overline{\mathbf{E}}\left(\vec{\beta}^{(m)}, 1, \ldots,|X|\right) .
$$

It follows from Lemmas 10 and 11 that for each $n \leq 2^{m}$ resp. $k<2^{m}, B_{m}$ includes a greedy order-ideal resp. greedy semi-ideal of size $n$ resp. $k$; this observation will be relevant in the following proofs.

The following lemma is presented not only for its own interest; it will be used in the next section.

Lemma 12. Let $X$ and $Y$ be order-ideals of $B_{m}$ such that $|X|=|Y|$ and $Y$ is greedy. Then $h(X) \leq h(Y)$, i.e., $\overline{\mathbf{h}}(X) \leq \overline{\mathbf{h}}(Y)$.

Proof. We prove the lemma via induction on $m$. We can assume that $m \geq 2$. Let $X$ be an order-ideal of $B_{m}$. It suffices to construct a greedy order-ideal $U$ such that $|U|=|X|$ and $h(X) \leq h(U)$. We can (and often will) change $X$ during the proof so 
that $h(X)$ does not decrease and $|X|$ remains the same. Now, for later reference, we formulate four facts; they are evident consequences of definitions. If, for some $k, Y$ is a greedy order-ideal of $B_{k}$ and $Z$ is an order-ideal of $B_{k}$ then

(Fact 1) $|Y| \geq 2^{k-1}$ implies that $Y$ contains a coatom of $B_{k}$;

(Fact 2) $|Y| \leq 2^{k-1}$ implies $Y \subseteq \downarrow c$ for some coatom $c$ of $B_{k}$;

(Fact 3) if $Z$ contains a coatom $d^{\prime} \in B_{k}$ and $Z \cap \uparrow d$ is a greedy order-ideal of $\uparrow d$ then $Z$ is a greedy order-ideal of $B_{k}$ (here $d=d^{\prime \prime}$ is an atom);

(Fact 4) $1 \leq j \leq|Y|$ implies that $B_{k}$ has a $j$-element greedy order-ideal which is a subset of $Y$.

Now, if there is an atom $a \in B_{m} \backslash X$ then $X$ is an order-ideal in $\downarrow a^{\prime}$, a Boolean lattice with $m-1$ atoms, whence the induction hypothesis yields an appropriate $U$ in $\downarrow a^{\prime}$, which is a greedy order-ideal in $B_{m}$ as well. Hence we can assume that $X$ contains all the atoms of $B_{m}$.

Let $a \in B_{m}$ be an atom. Since $B_{m}$ is a disjoint union of $\uparrow a$ and $\downarrow a^{\prime}, X$ is the disjoint union of $G^{\prime}:=X \cap \downarrow a^{\prime}$ and $G:=X \cap \uparrow a$. (Figure 2, where $G^{\prime}=G_{2}^{\prime} \cup \downarrow p$, depicts a particular but important case.) By the isomorphism theorem of intervals,

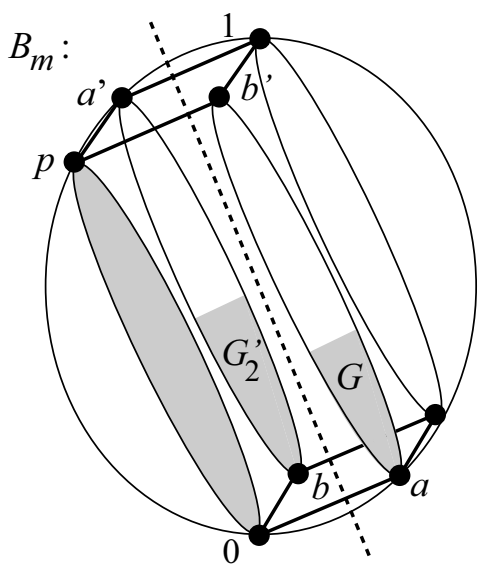

Figure 2

$$
\varphi: \downarrow a^{\prime} \rightarrow \uparrow a, \quad x \mapsto x \vee a \quad \text { and } \quad \psi: \uparrow a \rightarrow \downarrow a^{\prime}, x \mapsto x \wedge a^{\prime}
$$

are reciprocal isomorphisms. We know $1 \leq|G|$ from the latest assumption. The injectivity of $\psi$ and the fact that $\psi(x) \leq x \in X$ gives $\psi(x) \in G^{\prime}$ for any $x \in G$ show that $|G| \leq\left|G^{\prime}\right|$.

We can assume that $G^{\prime}$ resp. $G$ is a greedy order-ideal of $\downarrow a^{\prime}$ resp. $\uparrow a$ such that $\psi(G) \subseteq G^{\prime}$. (Then $G \cup G^{\prime}$ is necessarily an order-ideal, so it is still denoted by $X$.) Indeed, if this is not the case then, instead of $G$ and $G^{\prime}$, we could use $H$ and $H^{\prime}$ obtained as follows. First the induction hypothesis allows us to replace $G^{\prime}$ by a greedy order-ideal $H^{\prime}$ of $\downarrow a^{\prime}$ with $\left|G^{\prime}\right|=\left|H^{\prime}\right|$ and $h\left(G^{\prime}\right) \leq h\left(H^{\prime}\right)$. Clearly, $\varphi\left(H^{\prime}\right)$ is a greedy order-ideal of $\uparrow a$, and Fact 4 gives a greedy order-ideal $H$ of $\uparrow a$ such that $|H|=|G|$ and $H \subseteq \varphi\left(H^{\prime}\right)$. Since $h(G) \leq h(H)$ by the induction hypothesis, $H$ and $H^{\prime}$ do the job.

There are three cases. First we assume that $2^{m-2} \leq|G|$ or $2^{m-1} \leq|X|$. We have $2^{m-1} \leq|X|$ in both cases, for $|G| \leq\left|G^{\prime}\right|$. By Fact 1 , there is an atom $q \in B_{m}$ 
such that $\downarrow q^{\prime} \subseteq X$. By the induction hypothesis, there exists a subset $Y$ with $|Y|=|X \cap \uparrow q|$ and $h(Y) \geq h(X \cap \uparrow q)$ such that either $Y$ is empty or it is a greedy order-ideal of $\uparrow q$. We can let $U=Y \cup \downarrow q^{\prime}$, which does the job by Fact 3 .

The case when $\left|G^{\prime}\right| \leq 2^{m-2}$ is even simpler. Indeed, $G^{\prime} \subseteq \downarrow p$ for some coatom $p$ of $\downarrow a^{\prime}$ by Fact 2. Let $q=\varphi(p)$, which is a coatom of $B_{m}$. Clearly, $X \subseteq \downarrow q$. Applying the induction hypothesis to $\downarrow q$ we obtain a greedy order-ideal $U$ in $\downarrow q$ with $h(X) \leq h(U)$ and $|X|=|U|$, and $U$ does the job in $B_{m}$, too.

So we are left with the case depicted in Figure 2; namely, let $|G|<2^{m-2}<\left|G^{\prime}\right|$ and $|X|<2^{m-1}$. By Fact 1 we can choose an atom $b \in \downarrow a^{\prime}$ such that its complement $p$ taken in $\downarrow a^{\prime}$ belongs to $G^{\prime}$. Notice that $p=a^{\prime} \wedge b^{\prime}=\psi\left(b^{\prime}\right)$. We can assume that $G \subseteq\left[a, b^{\prime}\right]$, for otherwise $G$ can be replaced by a $|G|$-element greedy order-ideal of $\left[a, b^{\prime}\right]$. Let $G_{2}^{\prime}=G^{\prime} \cap\left[b, a^{\prime}\right], i=\left|G_{2}^{\prime}\right|, j=|G|$, and, to prepare an application of Lemma 4 for $n=m-2$, let $u:=i+j$ and $v:=0$. Since $|X|<2^{m-1}, u<2^{m-2}$. Choose a $u$-element greedy order-ideal $H_{2}^{\prime}$ in $\left[b, a^{\prime}\right]$. Then, by Fact $3, U:=H_{2}^{\prime} \cup \downarrow p$ is a greedy order-ideal of $\downarrow a^{\prime}$, whence $U$ is a greedy order-ideal of $B_{m}$. Finally, Lemmas 4 and 10 yield $h(X)=h(\downarrow p)+h\left(G_{2}^{\prime}\right)+h(G)=h(\downarrow p)+\sigma\left(\vec{\gamma}^{(m-2)}, 1, i\right)+$ $\sigma\left(\vec{\gamma}^{(m-2)}, 1, j\right) \leq h(\downarrow p)+\sigma\left(\vec{\gamma}^{(m-2)}, 1, u\right)+\sigma\left(\vec{\gamma}^{(m-2)}, 1,0\right)=h(\downarrow p)+h\left(H_{2}^{\prime}\right)+0=$ $h(U)$.

Lemma 13. Assume that Frankl's conjecture holds over m-element base sets. Then $\overline{\mathbf{h}}(X) \leq \overline{\mathbf{E}}\left(\vec{\beta}^{(m)}, 1, \ldots,|X|\right)$ for each semi-ideal $X$ of $B_{m}$.

Proof. We prove the lemma via induction on $m$. Let $X$ be a semi-ideal of $B_{m}$. According to Lemma 11, it suffices to find a greedy semi-ideal $Y$ of $B_{m}$ such that $|X|=|Y|$ and $h(X) \leq h(Y)$. Part (3) of Lemma 9 allows us to fix an enumeration $a_{1}, \ldots, a_{m}$ of the atoms in $B_{m}$ such that with the notations

$$
X_{0}=X \cap \downarrow a_{m}^{\prime}, \quad X_{1}=\left(X \cap \uparrow a_{m}\right) \backslash\left\{a_{m}\right\}, \quad X_{2}=X \cap\left\{a_{m}\right\}
$$

we have $\left|X_{1}\right|+\left|X_{2}\right| \leq\left|X_{0}\right|$. Note that $X=X_{0} \cup X_{1} \cup X_{2}$ and $X_{i} \cap X_{j}=\emptyset$ for $0 \leq i<j \leq 2$.

Clearly, $X_{0}$ is a semi-ideal of $\downarrow a_{m}^{\prime} \cong B_{m-1}$. To see that $X_{1}$ is a semi-ideal of $\uparrow a_{m} \cong B_{m-1}$, let $x \in X_{1}$. Then there is an atom $p \in B_{m}$ with $[p, x] \subseteq X$. If $p \neq a_{m}$ then $p \vee a_{m}$ is an atom of $\uparrow a_{m}$ and $\left[p \vee a_{m}, x\right] \subseteq X_{1}$. If $p=a_{m}$ then for each atom $q$ of $\uparrow a_{m},[q, x] \subseteq X_{1}$. Therefore $X_{1}$ is indeed a semi-ideal of $\uparrow a_{m}$.

Now let $I_{1}, \ldots, I_{m-1}$ be the standard interval sequence of $\downarrow a_{m}^{\prime}$ determined by the enumeration $a_{1}, \ldots, a_{m-1}$ of its atoms. Similarly, let $J_{1}, \ldots, J_{m-1}$ be the standard interval sequence of $\uparrow a_{m}$ determined by the enumeration $a_{1} \vee a_{m}, \ldots, a_{m-1} \vee a_{m}$ of its atoms. Notice that the mappings

$$
\varphi_{j}: I_{j} \rightarrow J_{j}, x \mapsto x \vee a_{m} \quad \text { and } \quad \psi_{j}: J_{j} \rightarrow I_{j}, x \mapsto x \wedge a_{m}^{\prime}
$$

are reciprocal lattice isomorphisms for each $j \in\{1, \ldots, m-1\}$. In fact, they are the restrictions of the reciprocal isomorphisms

$$
\varphi: \downarrow a_{m}^{\prime} \rightarrow \uparrow a_{m}, \quad x \mapsto x \vee a_{m} \quad \text { and } \quad \psi: \uparrow a_{m} \rightarrow \downarrow a_{m}^{\prime}, \quad x \mapsto x \wedge a_{m}^{\prime} .
$$

Now let

$$
Y_{0}=I_{1} \cup \cdots \cup I_{k-1} \cup U
$$

be a greedy semi-ideal of $\downarrow a_{m}^{\prime}$ (of rank $k$ ) such that $\left|Y_{0}\right|=\left|X_{0}\right|$. Here $U$ denotes a greedy order-ideal of $I_{k}$. Since $\left|X_{1}\right| \leq\left|X_{0}\right|$, we can choose a greedy semi-ideal $Y_{1}$ 
of $\uparrow a_{m}$ such that $\left|Y_{1}\right|=\left|X_{1}\right|$ and $\psi\left(Y_{1}\right) \subseteq Y_{0}$ (cf. Fact 4 from the previous proof). Let $\ell$ be the rank of $Y_{1}$. Then $\ell \leq k$ and $Y_{1}$ is of the form

$$
Y_{1}=J_{1} \cup \cdots \cup J_{\ell-1} \cup V
$$

where $V$ is a greedy order-ideal of $J_{\ell}$. The induction hypothesis gives $h\left(X_{0}\right) \leq h\left(Y_{0}\right)$ and $h\left(X_{1}\right) \leq h\left(Y_{1}\right)$, whence $h(X) \leq h\left(Y_{0} \cup Y_{1} \cup X_{2}\right)$. Notice that

$$
K_{1}=I_{1} \cup J_{1}, K_{2}=I_{2} \cup J_{2}, \ldots, K_{m-1}=I_{m-1} \cup J_{m-1}, K_{m}=\left\{a_{m}\right\}
$$

is the standard interval sequence of $B_{m}$ associated with the enumeration $a_{1}, \ldots, a_{m}$ of its atoms.

First we consider the case when $k=\ell$; the situation for $k=\ell=3$ is outlined in Figure 3. Since $U$ is a (greedy) order-ideal of $I_{k}, V$ is an order-ideal of $J_{k}$ and

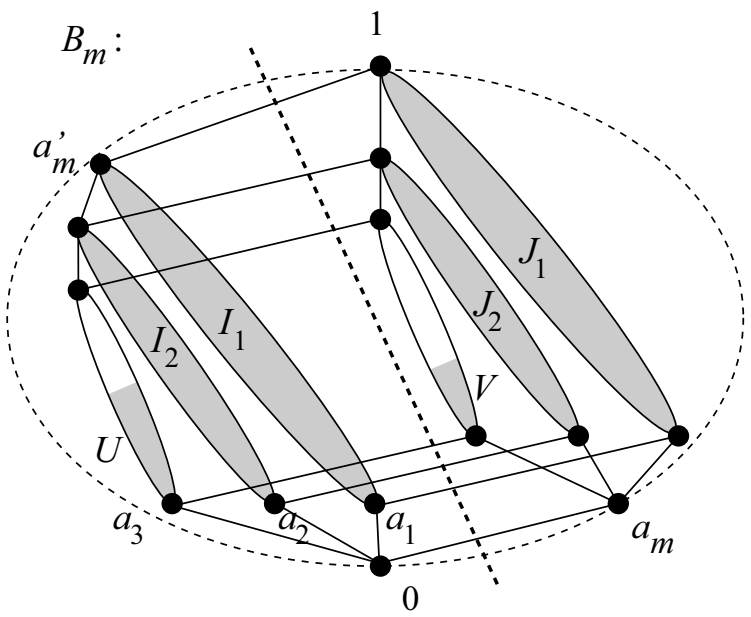

Figure 3

$\psi_{k}(V) \subseteq U$, we conclude that $U \cup V$ is an order-ideal of $K_{k}=I_{k} \cup J_{k}$. Let $W$ be a greedy order-ideal of $K_{k}$ such that $|W|=|U|+|V|=|U \cup V|$. We know from Lemma 12 that $h(U \cup V) \leq h(W)$. Clearly,

$$
Z:=\left(I_{1} \cup J_{1}\right) \cup \cdots \cup\left(I_{k-1} \cup J_{k-1}\right) \cup W=K_{1} \cup \cdots \cup K_{k-1} \cup W
$$

is a greedy semi-ideal of $B_{m}$, and we have $h\left(Y_{0} \cup Y_{1}\right) \leq h(Z)$. If $X_{2}=\emptyset$, i.e. $a_{m} \notin X$, then $|X|=|Z|$ and $h(X)=h\left(X_{0}\right)+h\left(X_{1}\right) \leq h\left(Y_{0}\right)+h\left(Y_{1}\right) \leq h(Z)$, as requested. In case of $a_{m} \in X$ we need an easy further step: let $Z^{+}$be a greedy semi-ideal of $B_{m}$ such that $Z \subseteq Z^{+}$and $\left|Z^{+}\right|=|Z|+1$. Then

$$
h(X)=h\left(X_{0}\right)+h\left(X_{1}\right)+h\left(a_{m}\right) \leq h(Y)+1 \leq h(Z)+1 \leq h\left(Z^{\prime}\right)
$$

together with $|X|=\left|Z^{+}\right|$settles the case $k=\ell$.

The general case is when $\operatorname{rank}\left(Y_{0}\right)=k \geq \ell=\operatorname{rank}\left(Y_{1}\right)$. This will be settled by an induction on $k-\ell$. For $k-\ell=0$ the job has already been done. Now assume that $k-\ell>0$; the situation for $(k, \ell)=(4,2)$ is outlined in Figure 4 . We are going to define a pair $\left(T_{0}, T_{1}\right)$ of greedy semi-ideals with the same properties as those assumed for $\left(Y_{0}, Y_{1}\right)$ such that $\operatorname{rank}\left(T_{0}\right)-\operatorname{rank}\left(T_{1}\right)<k-\ell$. 


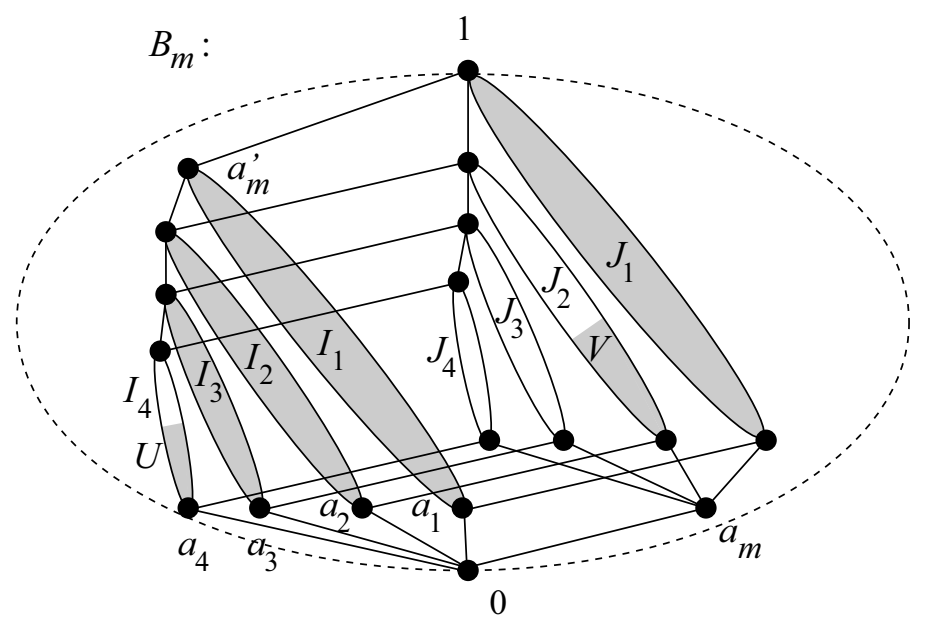

FIGURE 4

As an intermediate step we define a greedy semi-ideal $R_{0}$ of $\downarrow a_{m}^{\prime}$ and a greedy semi-ideal $R_{1}$ of $\uparrow a_{m}$. Let $i=|U|$ and $j=|V|$. In order to harmonize with the notations of Lemma 4 , define $u=i+j$ if $i+j \leq 2^{m-\ell-1}=\left|J_{\ell}\right|$ and let $u=\left|J_{\ell}\right|$ otherwise. Let $v=i+j-u$. Now let $R_{1}=J_{1} \cup \cdots \cup J_{\ell-1} \cup V^{+}$where $V^{+}$is a $u$-element greedy order-ideal of $J_{\ell}$. Let $U^{-}$be a $v$-element subset of $I_{k}$ such that $U^{-}$is a greedy order-ideal of $I_{k}$ when $v>0$. Then $R_{0}=I_{1} \cup \cdots \cup I_{k-1} \cup U^{-}$.

Finally, if $V^{+}=J_{\ell}$ and $U^{-} \neq \emptyset$ then let $T_{0}=R_{0} \backslash U^{-}$, and add $v=\left|U^{-}\right|$ many new elements to $R_{1}$ to obtain the greedy semi-ideal $T_{1}$ of $\uparrow a_{m}$. (These new elements will of course go into $J_{\ell+1}$.) In the other case when $V^{+} \subset J_{\ell}$ or $U^{-}=\emptyset$, we simply let $\left(T_{0}, T_{1}\right)=\left(R_{0}, R_{1}\right)$.

Clearly, we have $\left|Y_{0} \cup Y_{1}\right|=\left|T_{0} \cup T_{1}\right|$ and $\operatorname{rank}\left(Y_{0}\right)-\operatorname{rank}\left(Y_{1}\right)>\operatorname{rank}\left(T_{0}\right)-$ $\operatorname{rank}\left(T_{1}\right)$. So we are left with the duty of showing $h\left(Y_{0}\right)+h\left(Y_{1}\right) \leq h\left(T_{0}\right)+h\left(T_{1}\right)$.

It follows from Lemmas 4 and 10 that

$$
h_{I_{k}}(U)+h_{J_{\ell}}(V) \leq h_{I_{k}}\left(U^{-}\right)+h_{J_{\ell}}\left(V^{+}\right) .
$$

Therefore, measuring the total height in $B_{m}$ rather than in $I_{k}$ and $J_{\ell}$, we conclude

$$
h(U)+|U|+h(V)+2|V| \leq h\left(U^{-}\right)+\left|U^{-}\right|+h\left(V^{+}\right)+2\left|V^{+}\right|,
$$

which implies $h(U)+h(V) \leq h\left(U^{-}\right)+h\left(V^{+}\right)$, for $\left|U^{-}\right|+\left|V^{+}\right|=|U|+|V|$ and $|V| \leq\left|V^{+}\right|$. Therefore $h\left(Y_{0}\right)+h\left(Y_{1}\right) \leq h\left(R_{0}\right)+h\left(R_{1}\right)$. Finally, $h\left(R_{0}\right)+h\left(R_{1}\right) \leq$ $h\left(T_{0}\right)+h\left(T_{1}\right)$ is evident.

\section{THE END OF THE PROOF AND TWO CONJECTURES}

Now Theorem 1 follows from Lemmas 8, 11 and 13. Finally, Lemma 9 guarantees that Theorem 1 implies the Main Theorem.

We conclude the paper with two conjectures. Although they are formulated in terms of lattice theory, which goes well with the present paper, they will be translated to pure combinatorial language afterwards.

- For each semi-ideal $X$ of $B_{m}, \overline{\mathbf{h}}(X) \leq \overline{\mathbf{E}}\left(\vec{\beta}^{(m)}, 1, \ldots,|X|\right)$. 
- There is a function $f(m)$ such that $f(m) / 2^{m / 2}$ tends to $\infty$ and $\overline{\mathbf{h}}(X) \leq m / 2$ for every semi-ideal $X \subseteq B_{m}$ with $|X| \leq f(m)$.

According to Lemma 13 and Theorem 1, a positive solution of Frankl's conjecture would solve both problems in the affirmative. However, these conjectures might be easier (to prove or refute) than Frankl's one.

The ideas of Lemma 9 lead easily to the following combinatorial interpretation of the first conjecture. Given $1 \leq k \leq 2^{|A|}$, we want to find a union-closed family $\mathcal{F}, \emptyset \in \mathcal{F} \subseteq P(A)$, such that $\overline{\mathbf{w}}(\mathcal{F})$ be minimal. The conjecture asserts that we can obtain such an $\mathcal{F}$ by the obvious greedy algorithm in $2^{|A|}-k$ steps, starting from $\mathcal{F}_{0}=P(A)$ and deleting just one member of $\mathcal{F}_{i-1}$ in the $i$ th step. Note that Bošnjak and Marković [5] settle the first conjecture for $m \leq 11$.

A positive solution of the second conjecture would simply say that even if we cannot leave the assumption "Frankl's conjecture holds over $m$-element sets" out of the Main Theorem, the averaged Frankl's property holds for union-closed sets which are "essentially" larger than those treated in [7] (and mentioned in the Introduction.)

\section{REFERENCES}

[1] Tetsuya Abe: Excess of a lattice, Graphs and Combinatorics 18 (2002), 395-402.

[2] Tetsuya Abe: Strong semimodular lattices and Frankl's conjecture, Algebra Universalis 44 (2000), 379-382.

[3] Tetsuya Abe and Bumpei Nakano: Frankl's conjecture is true for modular lattices, Graphs and Combinatorics 14 (1998), 305-311.

[4] Tetsuya Abe and Bumpei Nakano: Lower semimodular types of lattices: Frankl's conjecture holds for lower quasi-semimodular lattices, Graphs and Combinatorics 16 (2000), no. 1, 1-16.

[5] I. Bošnjak and P. Marković: The 11-element case of Frankl's conjecture, Electron. J. Combin. 15 (2008), no. 1, Research Paper 88, 17 pp.

[6] E. Brown and T. P. Vaughan: Configurations with subset restrictions, J. Combin. Math. Combin. Comput. 48 (2004), 197-215.

[7] G. Czédli: On averaging Frankl's conjecture for large union-closed sets, Journal of Combinatorial Theory - Series A, to appear.

[8] K. Dohmen: A new perspective on the union-closed sets conjecture, Ars. Combin. 58 (2001), $183-185$.

[9] D. Duffus and B. Sands: An inequality for sizes of prime filters of finite distributive lattices, Discrete Math. 201 (1999), 89-99.

[10] M. El-Zahar: A graph-theoretic version of the union-closed sets conjecture, J. Graph Theory 26 (1997), 155-163.

[11] L. F. Fitina and J-C. Renaud: On union-closed sets and Conway's sequence, Bull. Austral. Math. Soc. 47 (1993), 321-332.

[12] P. Frankl: Extremal set systems. Handbook of combinatorics, Vol. 1, 2, 1293-1329, Elsevier, Amsterdam, 1995.

[13] Weidong Gao and Hongquan Yu: Note on the union-closed sets conjecture, Ars Combin. 49 (1998), 280-288.

[14] C. Herrmann and R. Langsdorf: Frankl's conjecture for lower semimodular lattices, http://www.mathematik.tu-darmstadt.de:8080/ herrmann/recherche/

[15] R. T. Johnson and T. P. Vaughan: On union-closed families. I, J. Combin. Th. Ser. A 84 (1998), 242-249.

[16] G. Lo Faro: Union-closed sets conjecture: improved bounds, J. Combin. Math. Combin. Comput. 16 (1994), 97-102.

[17] G. Lo Faro: A note on the union-closed sets conjecture, J. Austral Math. Soc. Ser. A 57 (1994), 230-236.

[18] P. Marković: An attempt at Frankl's conjecture, Publ. Inst. Math. (Beograd) (N.S.) 81(95) (2007), 29-43. 
[19] R. Morris: FC-families and improved bounds for Frankl's conjecture, European J. Combin. 27 (2006), 269-282.

[20] T. Nishimura and S. Takahashi: Around Frankl's conjecture, Sci. Rep. Yokohama Nat. Univ. Sect. Math. Phys. Chem. 43 (1996), 15-23.

[21] R. M. Norton and D. G. Sarvate: A note of the union-closed sets conjecture, J. Austral. Math. Soc. Ser. A 55 (1993) 411-413.

[22] B. Poonen: Union-closed families, J. Combinatorial Theory A 59 (1992), 253-268.

[23] D. Reimer: An average set size theorem, Combin. Probab. and Comput. 12 (2003), 89-93.

[24] J. Reinhold: Frankl's conjecture is true for lower semimodular lattices, Graphs and Combinatorics 16 (2000), 115-116.

[25] J-C. Renaud: Is the union-closed sets conjecture the best possible?, J. Austral Math. Soc. Ser. A 51 (1991), 276-283.

[26] J-C. Renaud: A second approximation to the boundary function on union-closed collections, Ars. Combin. 41 (1995), 177-188.

[27] J-C. Renaud and D. G. Sarvate: On the union-closed sets conjecture, Ars Combin. 27 (1989), 149-153.

[28] J-C. Renaud and D. G. Sarvate: Improved bounds for the union-closed sets conjecture, Ars Combin. 29 (1990), 181-185.

[29] I. Rival (ed): Graphs and Order, NATO Advanced Sci. Inst. Ser. C: Math. and Phys. Sciences 147, D. Reidel Publ. Co. Dordrecht-Boston (1985), p. 525.

[30] I. Roberts, Tech. Rep. No. 2/92, School Math. Stat., Curtin Univ. Tech., Perth, 1992.

[31] R. P. Stanley: Enumerative Combinatorics, Vol. I., Belmont, CA: Wadsworth and Brooks/Coole, 1986.

[32] T. P. Vaughan: Families implying the Frankl conjecture, European J. Combin. 23 (2002), $851-860$.

[33] T. P. Vaughan: A note on the union-closed sets conjecture, J. Combin. Math. Combin. Comput. 45 (2003), 95-108.

[34] T. P. Vaughan: Tree-sets in a union-closed family, J. Combin. Math. Combin. Comput. 49 (2004), 73-84.

[35] P. Winkler: Union-closed sets conjecture, Austral. Math. Soc. Gaz. 14 (1987), p. 99.

[36] P. Wójcik: Density of union-closed families, Discrete Math. 105 (1992), 259-267.

[37] http://www.math.uiuc.edu/ west/openp/unionclos.html

University of Szeged, Bolyai Institute, Szeged, Aradi vértanúk tere 1, HUngary 6720

E-mail address: czedli@math.u-szeged.hu

URL: http://www.math.u-szeged.hu/ czedli/

University of Szeged, Bolya Institute, Szeged, Aradi vértanúk tere 1, HUngary 6720

E-mail address: mmaroti@math.u-szeged.hu

URL: http://www.math.u-szeged.hu/ mmaroti/

Mathematical Institute of the Budapest University of Technology and Economics, Múngyetem RKP. 3, H-1521 Budapest, Hungary

E-mail address, E. T. Schmidt: schmidt@math.bme.hu

URL: http://www.math.bme.hu/ schmidt/ 